\title{
Planetary nebulae as tracers of the kinematic structure of the starburst galaxy IC 10
}

\author{
Denise R. Gonçalves ${ }^{1,2}$, Ana M. Teodorescu ${ }^{3}$, Alan Alves-Brito ${ }^{4}$, \\ Roberto H. Méndez ${ }^{3}$ and Laura Magrini ${ }^{5}$ \\ ${ }^{1}$ UFRJ - Observatório do Valongo, Ladeira Pedro Antonio 43, 20080-090 Rio de Janeiro, \\ Brazil. Email: denise@astro.ufrj.br \\ ${ }^{2}$ Department of Physics and Astronomy, University College London, Gower Street, WC1E \\ 6BT London, UK \\ ${ }^{3}$ Institute for Astronomy, University of Hawaii, 2680 Woodlawn Drive, HI 96822 Honolulu, \\ USA. Email: mendez@ifa.hawaii.edu; ana@ifa.hawaii.edu \\ ${ }^{4}$ PUC, Departamento de Astronomia y Astrofísica, Av. Vicuña Mackenna 4860, 782-0436 \\ Santiago, Chile. Email: abrito@astro.puc.cl \\ ${ }^{5}$ INAF - Osservatorio Astrofisico di Arcetri, Largo E. Fermi 5, I-50125 Firenze, Italy. \\ Email: laura@arcetri.astro.it
}

\begin{abstract}
The Local Group dwarf irregular galaxy IC 10 is the nearest starburst galaxy (at $725 \mathrm{kpc}$ ) and presents extended and complex HI kinematics. It is believed that IC 10 is still experiencing an intense and very recent burst of star formation, likely triggered by infalling gas from an extended envelope which is counter-rotating with respect to the galaxy proper rotation. In this contribution we summarize our preliminary results (Gonçalves et al. 2011, in prep.) obtained by performing a kinematic analysis of the PN population of IC 10. Our aim is to explore the kinematic connection of its intermediate to old stellar populations with that obtained from HI observations. Some PNe were found at large galactocentric distances and could therefore represent a trace of past tidal interactions.
\end{abstract}

Keywords. galaxies: dwarf, galaxies: kinematics and dynamics, galaxies: individual (IC 10), Local Group, planetary nebulae: general

\section{PNe vs. H I Radial Velocities in IC 10}

We have studied about $35 \mathrm{PNe}$ in IC 10, including newly discovered objects plus some PNe studied in Magrini et al. (2003). We used an [OIII] $\lambda 5007$ on-band/off-band technique with the FOCAS camera, at Subaru. The radial velocities (RVs) were measured for most of the detected sources, using a slit-less method (Méndez et al. 2009) by means of images taken through both the on-band filter and an echelle grism in three fields of 6.5 arcmin (Fig. 1a). The bins of PN RVs (black circles) are shown in Fig. 1b. In Fig. 1b we show our PNe radial velocities (black circles), whose average value of $-335 \pm 28 \mathrm{~km} \mathrm{~s}^{-1}$ agrees well with IC 10's radial velocity $\left(-348 \mathrm{~km} \mathrm{~s}^{-1}\right.$, NED).

Wilcots \& Miller (1998) (WM98) studied the H I kinematics of IC 10. By fitting a tilted ring model to the velocity field of IC 10 disk these authors found a rotation curve (their Fig. 5) that suggests a $\mathrm{V}_{\text {rot }}$ of $30 \mathrm{~km} \mathrm{~s}^{-1}$. In Fig. $1 \mathrm{~b}$ we compare the $\mathrm{PN}$ radial velocities with those obtained by WM98 from HI observations. In these velocity maps, the grey scale is indicative of the HI column density. The sense of the rotation is that the West side of the disk is approaching. WM98 conclude that IC 10 has a normal rotating disk, but with an abnormal counter rotation of its external envelope. Thus, Fig. 1b suggests that the PN kinematics follow that given by the HI gas. It is worth emphasising that, 


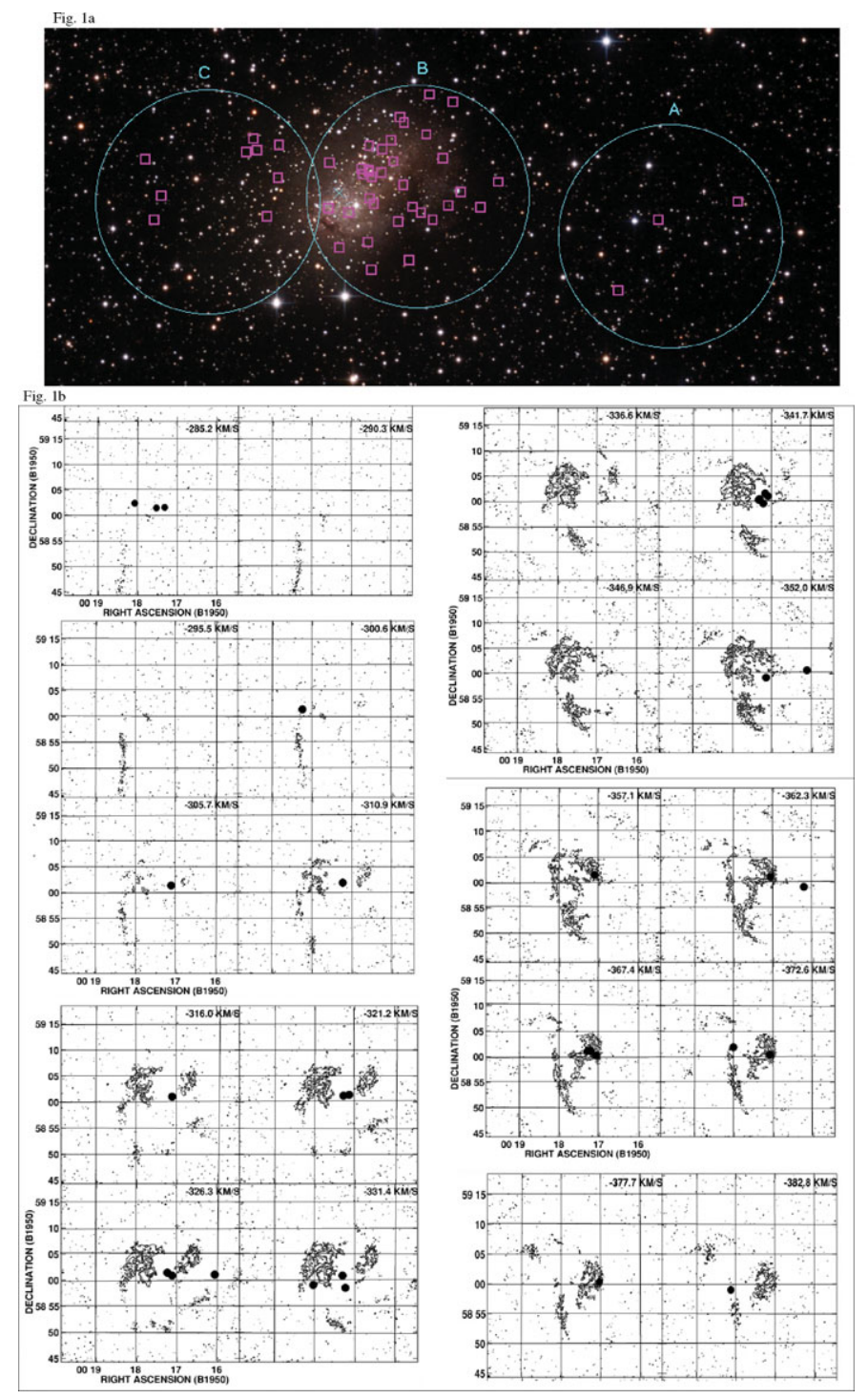

Figure 1. a) The three 6.5 arcmin FOCAS fields with the PNe we observed. b) IC 10's position fields per velocity bin with our PNe (black circles) superposed to the HI results of WM98.

while our study covers $\sim 3 \mathrm{kpc}$ from the galaxy's centre, the results based on H I are confined within less than $1 \mathrm{kpc}$ from the IC 10's centre. Our and the H I results suggest a galaxy formation and evolution scenario that might account for an inner normal rotating disk and with counter rotating gas further out. Interestingly, the IC 10 stellar population traced by $\mathrm{PNe}$ has a much larger extent than its present-time optical disk. The three kinematically confirmed PNe in IC 10's Field A tell us that the star formation in the past was extending towards larger radii than at present.

\section{References}

Magrini L., Corradi R. L. M., Greimel R., Leisy P., Lennon D. J., et al. 2003, A\&SA, 407, 51

Méndez R. H., Teodorescu A. M., Kudritzki R. P., \& Burkert A. 2009, ApJ, 691, 228

Wilcots E. M. \& Miller B. W. 1998, AJ, 116, 2363 